\title{
Are Bloating and Abdominal Distention Attributed to Gas Production and Visceral Sensitivity in Irritable Bowel Syndrome?
} (Am J Gastroenterol 2013;108:1516-1525)

\section{Chang Hwan Choi}

Department of Internal Medicine, Chung-Ang University College of Medicine, Seoul, Korea

\section{Summary}

Bloating and abdominal distension are common symptoms in patients with irritable bowel syndrome (IBS). Bloating is considered as a subjective sensation and abdominal distention as an objective sign, i.e., the visible increase in abdominal girth. ${ }^{1,2}$ They can be related to each other or not.

Recently, Zhu et al ${ }^{3}$ evaluated the effects of dietary factors on abdominal bloating and distention and the underlying pathophysiology in IBS. More specifically, they assessed the effects of gas production and visceral hypersensitivity on gastrointestinal (GI) symptoms after lactose ingestion in a population with lactase deficiency. IBS patients $(\mathrm{n}=277)$ and healthy controls $(\mathrm{HCs}, \mathrm{n}$ $=64$ ) underwent a $20 \mathrm{~g}$ lactose hydrogen breath test (LHBT) for evaluation of hydrogen gas production and lactose intolerance (LI) symptoms. Cumulative breath hydrogen excretion was quantified by measuring areas under the curve during LHBT. Five digestive symptoms characteristic of LI (bloating, borborygmi, nausea, pain and diarrhea) were recorded at 15-minute in- tervals on a scale of $0-4$ for 3 hours. Subjects who reported more than one point rise in LI symptoms during the LHBT were diagnosed as LI. Abdominal girth (199 IBS, 40 HCs) was measured in the standing and supine position, before and after LHBT, to evaluate the abdominal distension, using an inextensible metric tape measure over the umbilicus. Abdominal distention was defined to be increased by a minimum distention level of $\geq 0.5 \mathrm{~cm}$. Rectal sensitivity (74 IBS, 64 HCs) was assessed by barostat studies and visceral hypersensitivity was defined as below the 5.0 percentile limit for sensory threshold to discomfort/pain obtained from HCs. In the results, cumulative hydrogen excretion (AUC) during LHBT showed no difference between the groups $(P=$ $0.485)$ and the prevalence of abdominal distention after lactose ingestion (standing: $58 \%$ vs. $72.5 \%, P=0.083$; supine: $65 \%$ vs. $75 \%, P=0.213)$ was similar. In contrast, LI was more frequent in IBS (53.8 vs. $28.1 \%, P<0.001$ ), especially bloating (39.0 vs. $14.1 \%, P<0.001)$ and borborygmi (39.0 vs. $21.9 \%, P=$ 0.010 ). Only $59.0 \%$ of patients with bloating had distention. Hydrogen production as well as bloating score was not associated with girth increment. In the multivariate analysis for the factors

Received: September 18, 2013 Revised: None Accepted: September 18, 2013

(c) This is an Open Access article distributed under the terms of the Creative Commons Attribution Non-Commercial License (http://creativecommons. org/licenses/by-nc/3.0) which permits unrestricted non-commercial use, distribution, and reproduction in any medium, provided the original work is properly cited.

*Correspondence: Chang Hwan Choi, MD, PhD

Department of Internal Medicine, Chung-Ang University College of Medicine, 102, Heukseok-ro, Dongjak-gu, Seoul 156-755, Korea

Tel: +82-2-6299-1418, Fax: +82-2-6299-2017,E-mail: gicch@cau.ac.kr

Financial support: None.

Conflicts of interest: None. 
associated with LI symptom severity, hydrogen production increased bloating (odds ratio [OR], 2.19; 95\% confidence interval $[\mathrm{CI}], 1.09-4.39 ; P=0.028)$ and borborygmi (OR, 12.37; 95\% CI, 3.34-45.83; $P<0.001)$ but not distention $(P=0.673)$. Visceral hypersensitivity was associated with bloating (OR, 6.61; 95\% CI, 1.75-25.00; $P=0.005)$ and total symptom score (OR, 3.78; 95\% CI, 1.30-10.99; $P=0.014)$. The authors concluded that hydrogen gas production and visceral hypersensitivity both contribute to bloating and borborygmi, in IBS patients after lactose ingestion. Objective abdominal distention is not correlated with subjective bloating as well as gas production.

\section{Comments}

Bloating and abdominal distension occur commonly, especially in the functional GI disorders. In a population-based study in Olmsted County in the United States, the overall prevalence for bloating was $19 \%$ and $9 \%$ for abdominal distension. ${ }^{4}$ In a US study of an academic university clinic, $76 \%$ of 542 patients with IBS reported abdominal bloating. ${ }^{5}$

Bloating and distension have been attributed to excessive intestinal gas accumulation. ${ }^{6,7}$ But, the role of intestinal gas production as a cause of bloating and distention is controversial to date. Some reported increased hydrogen excretion in IBS patients compared with $\mathrm{HCs}$, ${ }^{8,9}$ whereas others showed no difference. ${ }^{10,11}$ Also, many other studies do not support that excessive gas induces bloating or abdominal pain. In a study using argon washout technique, there was no difference in the accumulation of intestinal gas between patients with bloating and healthy subjects. ${ }^{12}$ The study using CT scans combined with imaging analysis software has shown that excess gas was not associated with abdominal bloating in most patients. ${ }^{13}$ Other proposed underlying mechanisms of bloating and distension include impaired gas handling, altered gut motility, abnormal abdominal-diaphragmatic reflex, weakness of abdominal wall musculature, visceral hypersensitivity, intraluminal contents, food intolerance and carbohydrate malabsorption, small intestinal bacterial overgrowth, altered gut microflora and psychological factors. ${ }^{1,2,14}$ However, bloating and distention still remain to be incompletely understood and are considered challenging to treat in clinical practice.

To gain insight into the causes of bloating and distention, Zhu et $\mathrm{al}^{3}$ evaluated the effects of gas production after lactose ingestion and visceral hypersensitivity on bloating and abdominal distention, and the factors that increased the risk of these symptoms in IBS patients with lactase deficiency. Hydrogen gas pro- duction was similar between IBS and HCs and not associated with abdominal girth increase. It was only correlated with bloating and borborygmi scores in IBS. No similar correlation was found between gas production and symptoms in HCs. These findings indicate that not only gas production is associated with bloating and borborygmi, but also other factors must be present to lead to bloating in IBS patients, especially visceral hypersensitivity, and those might be more important than intestinal gas increment. Zhu et $\mathrm{al}^{3}$ found that visceral hypersensitivity was more frequent in IBS patients and significantly associated with bloating and borborygmi. However, there was no significant correlation between rectal sensory thresholds and LI symptom severity, so further studies were recommended to determine the relationship between visceral hypersensitivity and bloating. In addition, impaired intestinal gas handling also may be assessed as one of the underlying mechanisms of bloating that was not dealt in this study.

In this study, abdominal distention was present in more than half of each group (IBS and HCs); however, consistent with previous studies, ${ }^{4,15}$ there was no association between distension and gas production or bloating. These findings indicate that mechanisms of abdominal distention may be different from those of bloating. ${ }^{1,16}$ Redistribution of abdominal contents due to diaphragmatic descent and abnormal relaxation of the abdominal wall, that is to say abnormal viscero-somatic reflexes, might be associated with distention rather than excessive retention of intestinal gas. ${ }^{17,18}$

This study was prospectively conducted in a large cohort of IBS patients and HCs using a validated dietary intervention on bloating and distension, but has also several limitations, some of that were addressed in the paper. First, all subjects did not undergo every physiological measurement, especially rectal sensory thresholds. Second, diarrhea-predominant IBS patients made up $80 \%$ and non-constipated IBS $96 \%$ of the whole cohort, so these results may not be relevant to other subtypes, in particular constipation-predominant IBS. Also, this study was performed mainly in IBS patients with lactase deficiency. So, it may not be generalized to all IBS patients and further studies are needed to investigate the effects of gas production and visceral hypersensitivity on bloating and distention in IBS patients without lactase deficiency. Third, only hydrogen gas was assessed as a measure of carbohydrate malabsorption; measurement of other gases (e.g., methane) that may cause bloating and distension is also needed.

In conclusion, this study showed that both gas production 
and visceral hypersensitivity contribute to bloating and borborygmi, but not distention. The authors suggested the roles of diets on bloating and abdominal distention, and the hydrogen gas and visceral hypersensitivity as the underlying mechanisms of those symptoms in IBS. Although this study has several limitations, these results can be applicable to functional GI symptoms associated with diets in many IBS patients, and may guide dietary and medical managements.

\section{References}

1. Agrawal A, Whorwell PJ. Review article: abdominal bloating and distension in functional gastrointestinal disorders - epidemiology and exploration of possible mechanisms. Aliment Pharmacol Ther 2008; 27:2-10.

2. Schmulson M, Chang L. Review article: the treatment of functional abdominal bloating and distension. Aliment Pharmacol Ther 2011; 33:1071-1086.

3. Zhu Y, Zheng X, Cong Y, et al. Bloating and distention in irritable bowel syndrome: the role of gas production and visceral sensation after lactose ingestion in a population with lactase deficiency. Am J Gastroenterol 2013;108:1516-1525.

4. Jiang X, Locke GR 3rd, Choung RS, Zinsmeister AR, Schleck CD, Talley NJ. Prevalence and risk factors for abdominal bloating and visible distention: a population-based study. Gut 2008;57:756-763.

5. Chang L, Lee OY, Naliboff B, Schmulson M, Mayer EA. Sensation of bloating and visible abdominal distension in patients with irritable bowel syndrome. Am J Gastroenterol 2001;96:3341-3347.

6. Jones MP. Bloating and intestinal gas. Curr Treat Options Gastroenterol 2005;8:311-318.

7. Serra J, Azpiroz F, Malagelada JR. Mechanisms of intestinal gas retention in humans: impaired propulsion versus obstructed evacuation. Am J Physiol Gastrointest Liver Physiol 2001;281:G138-
G143.

8. Pimentel M, Chow EJ, Lin HC. Normalization of lactulose breath testing correlates with symptom improvement in irritable bowel syndrome: a double-blind, randomized, placebo-controlled study. Am J Gastroenterol 2003;98:412-419.

9. Kumar S, Misra A, Ghoshal UC. Patients with irritable bowel syndrome exhale more hydrogen than healthy subjects in fasting state. J Neurogastroenterol Motil 2010;16:299-305.

10. Gupta D, Ghoshal UC, Misra A, Choudhuri G, Singh K. Lactose intolerance in patients with irritable bowel syndrome from northern India: a case-control study. J Gastroenterol Hepatol 2007;22:22612265.

11. Farup PG, Monsbakken KW, Vandvik PO. Lactose malabsorption in apopulation with irritable bowel syndrome: prevalence and symptoms. A case-control study. Scand J Gastroenterol 2004;39:645649.

12. Lasser RB, Bond JH, Levitt MD. The role of intestinal gas in functional abdominal pain. N Engl J Med 1975;293:524-526.

13. Accarino A, Perez F, Azpiroz F, Quiroga S, Malagelada JR. Intestinal gas and bloating: effect of prokinetic stimulation. Am J Gastroenterol 2008;103:2036-2042.

14. Azpiroz F, Malagelada JR. Abdominal bloating. Gastroenterology 2005;129:1060-1078.

15. Houghton LA, Lea R, Agrawal A, Reilly B, Whorwell PJ. Relationship of abdominal bloating to distention in irritable bowel syndrome and effect of bowel habit. Gastroenterology 2006;131: 1003-1010.

16. Agrawal A, Houghton LA, Lea R, Morris J, Reilly B, Whorwell PJ. Bloating and distention in irritable bowel syndrome: the role of visceral sensation. Gastroenterology 2008;134:1882-1889.

17. Accarino A, Perez F, Azpiroz F, Quiroga S, Malagelada JR. Abdominal distention results from caudo-ventral redistribution of contents. Gastroenterology 2009;136:1544-1551.

18. Tremolaterra F, Villoria A, Azpiroz F, Serra J, Aguadé S, Malagelada JR. Impaired viscerosomatic reflexes and abdominal-wall dystony associated with bloating. Gastroenterology 2006;130:1062-1068. 